\title{
Erratum: Next-to-leading order calculation for jets defined by a maximized jet function [Phys. Rev. D 91, 034001 (2015)]
}

\author{
Tom Kaufmann, Asmita Mukherjee, and Werner Vogelsang
}

(Received 20 March 2020; accepted 23 March 2020; published 8 April 2020)

DOI: $10.1103 /$ PhysRevD.101.079902

Subsequent theoretical work (see, e.g., $[1,2]$ and references therein) has shown that single-inclusive jet cross sections may be formulated in terms of suitable partonic jet functions that depend on the jet algorithm under consideration. As was further pointed out [3], these jet functions are subject to momentum sum rules. Reference [4] showed that in the case of cone-based jet algorithms, previously available next-to-leading order (NLO) jet functions did, however, not obey these momentum sum rules. This was traced back to a mistake in the previous computations of the jet functions, and corrected jet functions were given.

We have noticed that our NLO results for jet cross sections for the class of $J_{E_{T}}$ algorithms presented in this paper are affected in a similar way and need to be corrected as well. The corrections are as follows:

(i) The upper limit on the jet mass used for $d \sigma_{j(k)}$, given in Eq. (21), is incorrect. It needs to be replaced by

$$
m^{2}<\frac{\left(P_{\mathrm{J}}^{\perp}\right)^{2}}{\beta z_{0}^{2}} \min \left(1-\left(1-z_{0}\right)^{n}, 1-z_{0}^{n}\right)
$$

where $z_{0}=1-v+v w$. This coincides with the expression given in Eq. (21) of our paper only for $z_{0}>1 / 2$. If instead $z_{0}<1 / 2$, the jet will be defined by parton $k$, which amounts to the condition $J^{(n)}\left(P_{\mathrm{J}}=p_{k}\right)<J^{(n)}\left(P_{\mathrm{J}}+p_{j}\right)$, as compared to Eq. (19). This condition leads to the contribution for $z_{0}<1 / 2$ in (1). We note that the new mass limit in Eq. (1) now essentially coincides with the one derived for the case of $d \sigma_{j k}$ in Eq. (12) of our paper, except for the trivial rescaling $P_{\mathrm{J}}^{\perp} \rightarrow P_{\mathrm{J}}^{\perp} / z_{0}^{2}$.

(ii) As a consequence, Eq. (22) should be replaced by

$$
\frac{d \sigma_{j(k)}}{d v d w}=\frac{\alpha_{s}}{\pi} \mathcal{N}_{a b \rightarrow K}(v, w, \varepsilon) v P_{j K}^{<}\left(z_{0}\right)\left(-\frac{1}{\varepsilon}\right)\left(\frac{\left(P_{\mathrm{J}}^{\perp}\right)^{2}\left(1-z_{0}\right)}{\beta z_{0}}\right)^{-\varepsilon}\left(\min \left(1-\left(1-z_{0}\right)^{n}, 1-z_{0}^{n}\right)\right)^{-\varepsilon} .
$$

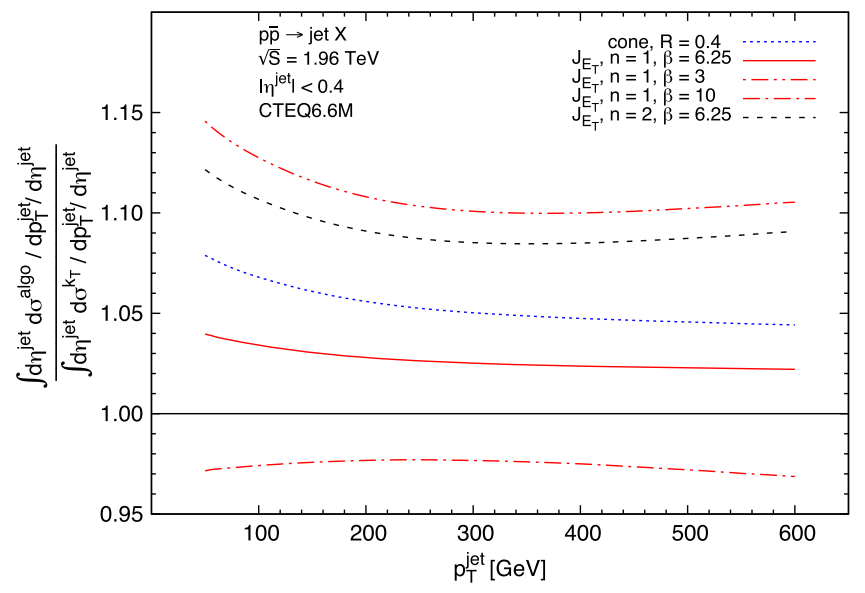

FIG. 1. Comparison of jet cross sections in the NJA for Tevatron kinematics. All results are shown relative to the one for the $k_{t}$ algorithm with $R=0.4$. 


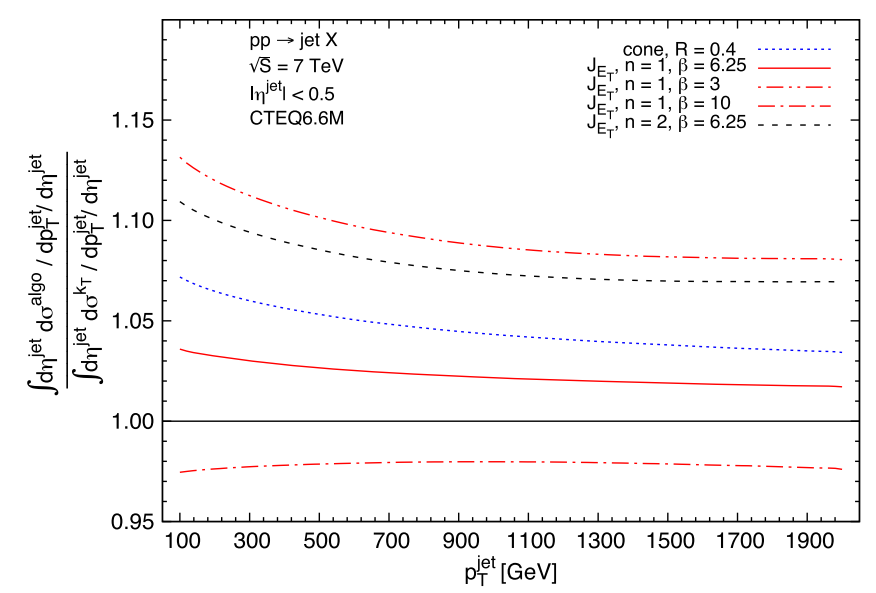

FIG. 2. Same as Fig. 1 but for $p p$ collisions at $\sqrt{S}=7 \mathrm{TeV}$.

(iii) Finally, Eq. (26) should be replaced by the simpler expression,

$$
d \sigma_{a b \rightarrow \mathrm{jet} X}=\mathcal{A}_{a b} \log \left(\frac{1}{\beta}\right)+\mathcal{B}_{a b}^{(n)}+\mathcal{O}(1 / \beta) .
$$

We note that more details of the derivations may be found in [5].

These corrections also affect the phenomenological results presented in Sec. III of our paper. In Fig. 1, we show the updated results for Tevatron kinematics, and in Fig. 2 we show the same for LHC kinematics. The other results for LHC kinematics with more forward rapidity bins differ in a similar way. The changes are overall numerically quite small, except for the cone algorithm that in the present paper was used only for illustrative purposes. All conclusions drawn from the phenomenological results remain unchanged.

\section{ACKNOWLEDGMENTS}

We are grateful to Zhongbo Kang and Felix Ringer for very useful discussions.

[1] T. Kaufmann, A. Mukherjee, and W. Vogelsang, Phys. Rev. D 92, 054015 (2015).

[2] Z. B. Kang, F. Ringer, and I. Vitev, J. High Energy Phys. 11 (2016) 155.

[3] L. Dai, C. Kim, and A. K. Leibovich, Phys. Rev. D 94, 114023 (2016).

[4] Z. B. Kang, F. Ringer, and W. J. Waalewijn, J. High Energy Phys. 07 (2017) 064.

[5] T. Kaufmann, Ph.D. thesis, University of Tübingen, 2017. 